\title{
Using Java for distributed computing in the Gaia satellite data processing
}

\author{
William O'Mullane ${ }^{1}$, Xavier Luri ${ }^{2}$, Paul \\ Parsons $^{3}$, Uwe Lammers ${ }^{1}$, John Hoar ${ }^{1}$, Jose \\ Hernandez ${ }^{1}$
}

the date of receipt and acceptance should be inserted later

\begin{abstract}
In recent years Java has matured to a stable easy-to-use language with the flexibility of an interpreter (for reflection etc.) but the performance and type checking of a compiled language. When we started using Java for astronomical applications around 1999 they were the first of their kind in astronomy. Now a great deal of astronomy software is written in Java as are many business applications.

We discuss the current environment and trends concerning the language and present an actual example of scientific use of Java for high-performance distributed computing: ESA's mission Gaia. The Gaia scanning satellite will perform a galactic census of about 1000 million objects in our galaxy. The Gaia community has chosen to write its processing software in Java. We explore the manifold reasons for choosing Java for this large science collaboration.

Gaia processing is numerically complex but highly distributable, some parts being embarrassingly parallel. We describe the Gaia processing architecture and its realisation in Java. We delve into the astrometric solution which is the most advanced and most complex part of the processing. The Gaia simulator is also written in Java and is the most mature code in the system. This has been successfully running since about 2005 on the supercomputer "Marenostrum" in Barcelona. We relate experiences of using Java on a large shared machine.

Finally we discuss Java, including some of its problems, for scientific computing.
\end{abstract}

PACS PACS 07.05.Kf · PACS 95.10.Jk · PACS 07.87.+v

\section{Introduction}

\subsection{The Gaia Mission}

The Gaia satellite is destined for the Lagrange point L2 early in 2012 after launch summer 2012 from French Guiana aboard a Soyuz Fregat Rocket. Gaia is the European Space Agency's (ESA) sixth cornerstone mission. Its goal to make a phase space map of

\footnotetext{
${ }^{1}$ European Space Astronomy Centre

${ }^{2}$ Departament d'astronomia i meteorologia, Universitat of Barcelona, ICC-UB/IEEC

${ }^{3}$ The Server Labs, Madrid, Spain
} 
our galaxy. Spinning around its own axis in a Lissajous orbit around L2 for five years gaia will continually scan the sky observing more than one thousand million $\left(10^{9}\right)$ celestial sources, on average eighty times each. The scientific goals of the experiment are manifold and covered in detail in (Perryman et al, 2001), the data will help key research in the composition and formation of our galaxy.

Gaia contains two astrometric telescopes at a fixed angle of $106.5^{\circ}$ as well as a radial velocity spectrograph and two photometers. The astrometric design allows true parallaxes (distances to stars) to be obtained (Makarov, 1998) after careful data processing. A least-squares fitting scheme named the Astrometric Global Iterative Solution (AGIS) (Lindegren et al, 2008) is currently foreseen to perform the astrometric data reduction. Equally involved processing is required for photometry and spectroscopy.

Gaia processing software is being written by the Gaia Data Processing and Analysis Consortium (DPAC). DPAC is a pan-European federation of institutes comprising of over four hundred astronomers, physicists and programmers. The consortium is led by eleven members of the community who form the DPAC Executive (DPACE). DPAC not only has the responsibility to write the processing software but also to run and maintain it until the final Gaia catalogue is produced around the year 2020. The software will be run in six data processing centres - each responsible for a different facet of the overall processing. An overview of DPAC and the processing is provided in (O'Mullane et al, 2007).

\subsection{Computing}

Simulations of Gaia data have been in production at the University of Barcelona (UB) since about 1998. Some of these simulations require considerable computing power and are discussed in Section 3 Initial AGIS experiments have been conducted using simulation data from UB in the past few years. The AGIS efforts are discussed in Section 2 By nature the data processing must be distributed. If one considers that there are $\approx 10^{12}$ (low resolution cutout) images downlinked from Gaia, at one millisecond per image that is over 30 years of processor time. Massive distribution is the only possibility to deal with this data. At this point the Gaia community has quite some experience with large software and computing efforts, which is not unusual in the science community. What some consider quite unusual is that all Gaia software is written in the Java language. We discuss the choice of Java in Section 4

\section{AGIS - a complex computing problem}

The Astrometric Global Iterative Solution (AGIS) provides the rigid framework for all of the Gaia measurements. Gaia is spinning freely and making observations which relate only to other observations made by Gaia. It is an absolute instrument - there is no list of input stars such as there was for Hipparcos (ESA, 1997), the predecessor of Gaia. What this means is that the Gaia data must be reduced in a self-consistent manner such that all individual observations of celestial sources, the model of each source's position and motion, Gaia's own attitude, orbit and velocity must be in harmony. Later the entire system will be aligned with the International Celestial Reference System (ICRS).

The complete mathematical formulation for AGIS is presented in (Lindegren et al, 2008) while the computational framework is described in (O'Mullane et al, 2008). Here 
we recap these aspects briefly in section 2.1 before discussing results (section 2.2) and performance/manpower trade offs (section 2.3).

\subsection{AGIS overview}

AGIS is a block-iterative solution for the Gaia astrometry. It consists of at least four blocks which may be calculated independently. Each block is however dependent on the result of the other blocks. We term one step through all blocks an outer iteration, although the term outer is frequently dropped. This is simplistically formulated in the following equations :

$$
\begin{aligned}
& S=A+G+C \\
& A=S+G+C \\
& G=S+A+C \\
& C=S+A+G
\end{aligned}
$$

which are discussed further below.

The vast majority of Gaia sources (Equation 1) may be described by a six parameter astrometric model. These parameters and their derivation are fairly standard in modern astrometry (Murray, 1983) and describe the position and motion of the source in three dimensions. The parameters are :

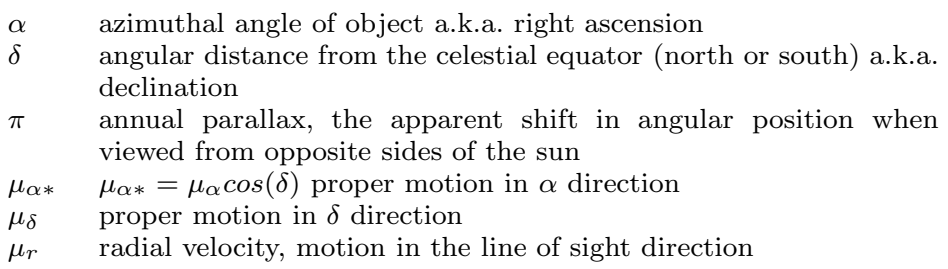

From the $\approx 80$ observations of each source made by Gaia a least-squares fitting of the observations to the model may be made. The fit must include several intricacies namely:

- the orientation of Gaia in space (or attitude, Equation 2)

- the path of light through the instrument (or calibration, Equation 4)

- global parameters such as relativistic numbers (Section 3)

- light bending according to general relativity (see (Klioner, 2003))

Current best estimates for attitude, calibration and global parameters are used for any given fit of the source. This fit must be performed on all sources but to make the problem tractable a subset of sources may be treated first. Around $10^{8}$ sources are needed to make the global reference frame but it could be as many as $5 \cdot 10^{8}$. Once this solution has converged the source calculation (using the final attitude etc.) may be performed on all remaining sources. This is an important efficiency improvement. A direct solution for a large number of sources has been shown to be intractable (Bombrun et al, 2010). 
The application of the source equation (known as source update) requires the gathering of all observation of the source and the current attitude, calibration and global parameters. The application of the relativistic model requires precise ephemerides of the planets and of the satellite. Our current approach is to group observations together on disk for easy loading, since there is no cross talk between the source equations i.e. all sources may be processed in parallel if we wish. The other data required is sufficiently small (order of a few hundred MB) that it may be loaded once in memory and used to process multiple sources, typically batches of several thousand sources per job on a processor.

Attitude and calibration on the other hand require all observations in given time periods. We do not need to hold on to the observations but may add their contributions to partial equations. Hence as each source is updated the updated parameters and the observations are used to update the partial solutions for the attitude and calibration. The system has been run with up to 100 computing nodes and 1400 Java threads. We constantly encounter bottlenecks usually in the calibration and attitude processes for the obvious reason that they need to see all sources. The processing of several thousand sources in a job makes the communication more efficient - we send fewer large messages rather than millions of small ones.

\subsection{Results}

AGIS has been running at ESAC since early 2006 with increasing numbers of sources and observations. This is discussed briefly in (O’Mullane et al, 2008) from which we reproduce table 1 below. Since the entire system is designed for AGIS and we have few interconnects between processes we did not find it necessary to use any HPC library nor GRID software. Effectively jobs are taken from a Whiteboard by numerous DataTrains which load the appropriate data and process it writing the results back to a database. The Whiteboard may simply be considered as a table in a database where jobs are written an updated. DataTrain is a term coined within DPAC for a process which runs through a set of data passing it to a series of algorithms. It provides an abstraction layer between algorithm and data access.

The machine used for the Gaia tests so far has been purchased in installments by ESA. The initial machine for the first tests in 2005 consisted of twelve nodes each with $6 \mathrm{~GB}$ of RAM and two processors (3.6GHz Xeon EM64T). An EMC storage area network (SAN) with 5 TB of disk is attached to the nodes using fibre optic cable and the nodes are also connected via Gigabit Ethernet cards in a local area network. AGIS has also been tested, and shown to run as fast, using a cheap Rack Server Network Attached Storage device of 6 TB. This cluster was upgraded to 18 nodes in 2006 and a further 4 nodes but with quad core processors were added in 2007. The quad core processors functioned very well for us providing the performance of 4 processors and in table 1 the number of processors counts each core as a processor.

This is not very special machine, it consists of standard Dell power edge blades with standard Intel Xeon processors running Red Hat Linux. The blades are housed in a standard Dell rack. There is no special HPC software used, a network of normal Linux machines could work in the same manner. As such this machine perhaps does not merit the term HPC but we will move toward 10 Teraflops in the coming years.

Various different types of astronomical test have been performed to show AGIS produces the correct solution for Gaia. For this paper we are mainly interested in per- 
Table 1 AGIS run times decrease as more processors are added. Note that the data volume increased from 2005 to 2006 from 18 months to 5 years, the processor power also increased but the run time went up. This was dramatically improved in 2007. In 2010 we have implemented a new math frame work around Conjugate gradient - this provides a better solution at some cost. The normalised column shows throughput per processor in the system (total observations/processors/hours) e.g. an indication of the real performance.

\begin{tabular}{|l|l|r|r|c|}
\hline Date & Observations & \multicolumn{1}{|c|}{ Procs } & \multicolumn{1}{c|}{ Time } & Normalised \\
\hline 2005 & $1.6 \times 10^{7} \mathrm{src}$ & 12 & $3 \mathrm{~h}$ & $0.9 \times 10^{6} \mathrm{obs} / \mathrm{hour}$ \\
\hline 2006 & $8 \times 10^{7}$ & 36 & $5 \mathrm{~h}$ & $0.5 \times 10^{6} \mathrm{obs} / \mathrm{hour}$ \\
\hline 2007 & $8 \times 10^{7}$ & 24 & $3 \mathrm{~h}$ & $1.3 \times 10^{6} \mathrm{obs} / \mathrm{hour}$ \\
\hline 2008 & $8 \times 10^{7}$ & 31 & $1 \mathrm{~h}$ & $3.2 \times 10^{6} \mathrm{obs} / \mathrm{hour}$ \\
\hline 2009 & $2.6 \times 10^{8}$ & 50 & $1.8 \mathrm{~h}$ & $2.8 \times 10^{6} \mathrm{obs} / \mathrm{hour}$ \\
\hline 2010 & $4 \times 10^{9}$ & 68 & 9.5 & $6.2 \times 10^{6} \mathrm{obs} / \mathrm{hour}$ \\
\hline
\end{tabular}

formance however, which is best presented by table1. We see that AGIS has been slow in 2007 but finally it was made much faster in 2008 with a prospect of more improvement to come. It should be noted that more functionality has also been introduced each year - a more complete source model, a more complex calibration scheme etc. but the efficiency has been increased. Some speedups are due to profiling while others are due to mathematical methods. Between the Lund group and the ESAC group finally the problem is being tackled from both math and computing side with competence. Early work on AGIS was not so fruitful as it treated the problem as something to just be run on a computer rather than a problem for which a system needed to be designed to make it efficient. In that system math and computing techniques have been questioned and varied to arrive at the efficient implementation we have today.

\subsection{Performance vs Manpower}

We know we could write some parts of AGIS to run perhaps two or more times faster than Java. We could get a little more performance by utilising special machines and rewriting our code again for example toward a cell processor hybrid such as the Roadrunner. Speed of code is indeed an issue for Gaia in general but manpower is perhaps a bigger issue - we have a great deal of processing software to write. The estimated manpower in man years for the Gaia processing is around 2000. Granted this includes operation but development alone pre launch is estimated at 1000 man years. In the case of the astrometric solution we have good records. The initial working AGIS in ESAC was done in about 180 man days so just over half a man year. It was clear to us that this was only possible in Java - in $\mathrm{C}$ or $\mathrm{C}++$ this would have taken far longer. This claim is of course difficult to quantify, as a programming collective we have decades of experience with $\mathrm{C}$ and $\mathrm{C}++$ projects and it is our opinion that coding in Java is cleaner and faster. Even in Java the problem is difficult. The previous solution which AGIS replaced was worked on for four years by various people; to be modest lets say only 8 man years went into it, but it was more like 12. To date on AGIS we have spent about 15 man years and we estimate to finish AGIS in Java we need another 10 man years with probably more after launch. There is also post launch maintenance to consider - we feel Java maintenance will be lower cost than say C, and we have eight years of operations to consider - we have 27 man years in the plan for this. 
Of course it is difficult to calculate but we would need far more manpower to manage code in $\mathrm{C}$ and more still if we were to customise for a particular computer system (especially as it would become obsolete during our operations). Again just for AGIS we are talking of over 50 man years of effort which in today's monetary terms is around 5 million euro. Even $20 \%$ more effort to code in another language would be a considerable sum. We think we can buy the hardware we need to run AGIS for around a million euro. It has been observed on other space programs than manpower typically ends up costing more than machines and apparently we are no different. Our intention is also to buy the most common and cheap processors - these are the the chips Java is usually running fastest on as well because of their ubiquity. Hence a special more super computer oriented machine would probably cost more. Finally there is power consumption if we could make AGIS 4 times faster we could save $25 \%$ on power. Energy costs may well go up but probably not faster than manpower costs since the saving on energy requires spending on manpower to customise code for our "hard to code" problem these will cancel each other out.

\section{Gaia Simulations - use of shared computing resource}

The development of the Gaia simulator has been an integral part of the Gaia data processing. This software tool is designed to provide realistic simulations of the Gaia observations, closely mimicking the format and content of the data that Gaia will send to ground. The main purpose of this simulated data has been the feeding of the data processing chain in order to test and validate it, although it has also been used for other purposes, for instance to evaluate some satellite design options and to prepare the mission scientific case.

As mentioned above, the simulator has been running at the University of Barcelona since 1998. Its initial development was closely tied to the first attempts to develop a viable Global Iterative Solution but now it has become a mature tool able to simulate a wide variety of celestial objects, physical and instrumental effects and data formats for the multiple data processing modules developed by the Gaia DPAC.

In this section we will first review our experience with the development in Java, the advantages and drawbacks we found, and then discuss a specific (and extreme) example of Java versus C performance found during this development.

\subsection{Java, a new kid on the block}

When the development of the simulator was started the first choice to be made was the programming language to use. The team undertaking this task was at the time (1998) mainly (and almost exclusively) composed of scientists, with few software engineering expertise. The obvious choice, based on the programming experience of the team, would have been FORTRAN with perhaps $\mathrm{C}$ as a second, but somewhat frowned upon, choice. Furthermore, astronomical and numerical libraries were available in FORTRAN, and in most cases also in C, but not in Java.

However, Java was finally chosen for two main reasons:

1. The ongoing Global Iterative Solution development contract was going to be implemented in Java by requirement of ESA. Given that the two projects were closely tied at the time using a common language was natural. 
2. The advice of professional software engineers from ESA and some members of the team with software engineering background pointed towards the use of an Object Oriented language, strongly advising against FORTRAN for a large collaborative project. This left the choice between $\mathrm{C}++$ and Java.

At the end Java was chosen, but not without frequent second thoughts in the coming years. The widening scientific community that was becoming involved in the project was reticent for some time to adopt the new language, specially given the accumulated experience in FORTRAN and $\mathrm{C}$ and the lack of libraries in Java.

However, eleven years later the landscape has much changed, and now Java has been even more widely (almost completely) adopted as a viable programming language for scientific programming in the Gaia simulator community. Furthermore, the project has become large and complex, involving development teams distributed around Europe. The management of the project has at this stage adopted many professional software development tools (UML, Hudson, PMD, Checkstyle, etc.) that would not have been available had a language like FORTRAN been chosen, making the coding much more robust and reliable. Also, like the team developing AGIS and based on previous experiences, they feel that the development has been quicker and more seamless than it would have been using $\mathrm{C}++$. The motives for this feeling are varied, but one of the big reasons is that thanks to the garbage collection implemented in the JVMs memory leaks are less of an issue. Since the simulator is quite intensive in memory usage, not having to worry about this problem is seen as a great advantage.

In short, the initial reticences on the use of Java for scientific programming in Gaia have vanished, to the point that one of the managers of the simulator development has changed the teaching of programming for first year physics students from FORTRAN to Java.

\subsection{The blessing of portability}

One of the advantages of Java not mentioned in the previous section is its portability. As said, the simulator has been running for eleven years and during this time it has been ported to several machines, and in the process Java has shown that its portability is real and practical.

Due to its intensive computation needs the Gaia simulator has mostly been run at supercomputing centers, using significant amounts of computation time It was initially run at the Centre de Supercomputació de Catalunya (CESCA) were several mediumsized clusters were used: an IBM SP2, a Compaq HPC320, an HP RX2600 and an SGI ALTIX 3700. In all cases the migration from one cluster to the next was seamless, the only serious complication being the adaptation of the execution to the different queue systems in each machine. Later on, in 2005, the simulator was moved to the Barcelona Supercomputing Center (BSC) where the Mare Nostrum supercomputer had been just installed. Again, the migration was quite simple, with the major complication being how to adapt the execution to the new distributed environment and queue system. Today the simulator is still running there, having cruised through a Mare Nostrum upgrade in 2006 without needing any significant change and having consumed a some millions of CPU hours and generated many terabytes of simulated data.

In these years the portability of Java has therefore been a major advantage, saving time and resources that otherwise would have been devoted to adapting the code to the 
new environment. Furthermore, the portability of Java has also allowed the running of the simulator on other environments like local clusters for testing and development and a cluster at CNES for tailored small simulations through a web page manager.

\subsection{Performance in scientific computation}

In the initial years of the project the performance in numerical computing of Java was discussed many times. Not having previous experience on its use for scientific computation there was some worry that the language could prove to be too slow at some point of the development. However, some initial tests with a set of some often-used numerical algorithms showed that Java was not much slower that $\mathrm{C}$ in solving these problems, thanks to the Just In Time (JIT) compilation in the Java virtual machines.

In the next years the JIT virtual machines steadily improved, and the first working code was developed in the project, showing in practice that the implementation in Java was not producing any of the feared bottlenecks. Nowadays, Java has become part of the landscape in scientific programming in Gaia, and although worries about performance are still occasionally an element of resistance, given the accumulated experience and clear savings in development time performance is no longer an issue.

Furthermore, the development model in DPAC is based on six-month development cycles; at the end of each cycle an improved version is produced and tested, leading to further requirements, corrections and improvements for the next cycle. In this framework the optimisation of the system is not as important as the maintainability and flexibility of the code to allow such a quick production. The robustness and clarity of Java helps a lot in such a process, and as mentioned before has probably saved a significant amount of money in programming resources.

A real example encountered during the simulator will illustrate this point. A key piece of the Gaia astrometry is the calculation of the so-called "relativistic corrections", the changes on the apparent position of the objects in the sky deriving from relativistic effects: aberration, gravitational light bending, etc. This is a complex calculation taking into account the ephemeris of the major solar system bodies and requiring (for a $\mu$ as accuracy) to reach the limit of the numerical precision of variables of the "double" type (64 bit floating point).

An initial (legacy) implementation of these calculations was available from S. Klioner in the form of $\mathrm{C}$ code and was used in the simulator until 2008 through JNI calls. At that time, in order to avoid the inconveniences of mixing two languages in the simulator, the same author developed for DPAC a new implementation coded in Java. Both implementations were thoroughly compared and results matched at sub$\mu$ as level. However, during the testing it was found that the computation times differed substantially between the two versions, the Java version being between 4 and 10 times faster than the $\mathrm{C}$ version! Possible external sources of this difference (like overheads of the JNI calls) were ruled out and it was concluded that the difference was actually intrinsic to the code.

It is possible, even likely, that an optimisation of the $\mathrm{C}$ code would make it much more efficient, to at least the level of the Java code. However, this example clearly shows how the same developer did a quicker and better job in Java (a language that, unlike $\mathrm{C}$, he was unfamiliar with). On the other hand, the difference possibly comes also from the excellent refactoring afforded by the new JITs virtual machines that 
now automatically makes many of the performance fixes which previously had to be manually implemented - in $\mathrm{C}$ of course all optimisations must be done by the coder.

Finally, the optimization work on the Java code has continued. The team is now exploring the increasing possibilities of the options for aggressive optimization and garbage collection tailoring available in some Java virtual machines (specially the IBM one, used in the MareNostrum supercomputer) that will possibly lead to further improvements in the performance of the simulator.

\section{Java, Gaia and commercial uses}

When (O’Mullane et al, 2000) was presented at the Astronomical Data Analysis and Software Systems (ADASS) conference in 1999 most people in that community did not know what Java was and this was the only paper mentioning Java. Two years later about half of the astronomy related projects at ADASS involved Java. Some project must take the first step, in this case Gaia and Planck were experimenting with Java. These experiments possibly made it easier for the Integral SOC and the entire Herschel Science Ground Segment to be written in Java (Wieprecht et al, 2004). Gaia and Java go back many years, the initial prototype for the Global Iterative Solution was already in Java in 1999 (O’Mullane and Lindegren, 1999).

Java in the late 90s was not the same as it is today but already then it was seen to have potential for science development. For a project like Gaia we were faced with a life span of over 20 years for our software and an entrenched Fortran community. It was clear already back then, as discussed earlier, that Gaia processing software would not be written in Fortran but needed to be in a more modern, preferably OO, language, $\mathrm{C}++$ being the obvious choice. The transition for some scientists from Fortran to $\mathrm{C}++$ was seen as difficult or impossible. Java simply worked more easily and was chosen and eventually accepted by all.

\subsection{Negative Aspects}

We do not claim Java is the silver bullet (F.P., 1987) and we have several problems which we live with especially in numerical coding. As already mentioned especially when we started, there were no good numerical libraries. The situation improved from about 2000 onwards when Java started to become really usable for scientific computing in terms of robustness and performance. A number of promising numerical library developments took place (e.g. JAMA - A Java Matrix Package) but until around 2005 most of them had stopped, leaving the distributions and code available but largely unmaintained. A positive exception is the Apache Common Math library which is still in active development and in extensive use in almost all of Gaia's data processing software. Early versions of Apache Common Math were missing linear algebra functionality needed by us but that has improved with time.

In parallel with diminishing efforts for developing numerical libraries we observed a general decline in support for Numerics in Java. For instance the Numerics Working Group of the Java Grande Forum (1), initially a driving force behind many positive developments around Java Numerics, has effectively ceased to exist. This is a bit worrying

\footnotetext{
1 http://math.nist.gov/javanumerics/
} 
along with a general perceived lack of support for Java in the traditional conservative supercomputing scene that in terms of languages remains to be dominated by Fortran and $\mathrm{C}$ until today.

In 1998 W. Kahan, one of the key persons behind the IEEE 754 Standard for Binary Floating-Point Arithmetic, delivered a keynote "How Java's Floating-Point Hurts Everyone Everywhere" Kahan and Darcy (1998) at a Java HPC workshop. In this contribution Java gets harshly criticized for for a number of IEEE non-compliances which could and should have been avoided by the language designers. In subsequent years Sun was repeatedly asked to rectify the known deficiencies but chose to ignore all complaints and even today those issues raised more than 10 years ago are, to our knowledge, still present in all existing Java implementations. With Java now under Oracle's control it seems unlikely that the situation will change in the foreseeable future. Fortunately, in our view most of the points are fairly subtle in nature and are unlikely to show up as perceivable flaws in "ordinary" numerical application code. We can confirm this for all of Gaia's software and in particular for the AGIS system described here in more detail (Sect. 2) with one exception: Java does not provide any means to use what IEEE 754 calls 'trap-handlers' for capturing and dealing with numerical exceptions like division by zero. Every arithmetic operation in Java always delivers a valid result and that can make the debugging of numerical code extremely difficult and time consuming. As an example, there was a coding error in the AGIS attitude update that caused the introduction of an NaN value into a large matrix of equations which then propagated and spread through the matrix before eventually leading to $\mathrm{NaN}$ in an end result much later. Without the option to have an exception raised at the first occurrence of an $\mathrm{NaN}$ condition the only way to find the problem is to check explicitly every intermediate result for NaN (Double.isNaN(x)). For obvious reasons this is not a viable option in a large numerical code.

\subsection{Features}

One of the things which made java work well for us was its built in features. We were especially happy to have multi threading and distributed programming such as RMI (Remote Method Invocation - allows calling a method on an object on another machine) built in to the language.

\subsection{Rise of Java in industry}

At the same time (back in the late 90's) we saw in industry a surge of Java programming as it pushed from the the Web language clearly into the back office. Enterprise Java Beans were starting to appear behind web pages accessing databases and wrapping legacy applications to make them network available. Java was no longer an "applet" language for making more interactive web pages - it was handling credit cards and transactions and doing serious work. The fact that Java is $100 \%$ portable to all O/Ss where the JVM has been ported to and backward compatible means that using libraries is no longer a painful issue.

Companies such as IBM, Oracle, BEA, Sun and many open source vendors have created Java application servers that support the Java standards in JavaEE produced by Sun. 
Since then frameworks to further aid productivity in the development of Java software such as Spring and Hibernate have appeared on the scene and these have helped with the adoption of Java as the technology of choice for developing software. In addition the fact that there are thousands of open source libraries available to use in Java projects has also helped the spread of Java.

\subsection{Maintainability, Robustness and Performance}

When developing software there are a number of key issues that have to be addressed including but not limited to Maintainability, Robustness, Scalability

Addressing maintainability first, Java compared to other languages such as C++ and Fortran offers a number of advantages. The defacto editor for Java, Eclipse, provides a number of features that makes creating and maintaining Java code easier; Graphical syntax highlighting, Refactoring wizards to improve the design of the application; Plugins to produce documentation. Java was also one of the first languages to have a really good implementation of the $\mathrm{xUnit}$ testing frameworks with the widely adopted JUnit. With Java 1.5 and the introduction of Generics and Annotations and with techniques such as Aspect Oriented Programming (AOP - not currently used on Gaia) we have the possibility of developing software with less code and the less code there is, the more maintainable it is.

Java is more robust than $\mathrm{C}++$, primarily because the $\mathrm{C}++$ component of all Java programs, the JVM itself, is the same for all Java programs and therefore tried and tested millions of times.

Many people believe that Java is not as scalable as other languages because of the overhead of the Java bytecode interpretation. However the Java Hotspot server compiler, especially the one in Java 1.6 , is incredibly efficient at optimising code that is called very frequently.

\subsection{Portability and the Cloud}

Although there are minor problems with Java portability this is usually in Graphical User Interfaces rather than in the type of code forming the majority of the Gaia environment. As stated in Section 3 our simulation code has been ported over many years to many platforms with little effort. Currently development is done on MacOs, Linux and windows systems without issue. Such portability lead us to consider using the Amazon E2C for the Astrometric solution (Section 2). We, unlike CERN Shiers (2009) are not yet tied in to one architecture. Although these days even CERN use some cloud resource.

With about 2 person weeks of effort part of our team (Parsons and Olias) got AGIS running on the cloud. Some problems were encountered with the database configuration, although at least Amazon already hand a VM with Oracle on it. Also a scalability problem in our own code was found and remedied - prior to this we had no opportunity to run 1400 threads. It worked well, at least as well as our cheap in house cluster. Hence we agree with the detractors of the Cloud - it is not a supercomputer with super fast interconnect, but then one is not paying super prices either.

In fact although our intention is/was to build a cheap cluster (around 1MEuro) for AGIS we estimate all AGIS mission processing for 100 Million Stars could be done for 
about 400KEuro. When energy is factored in this makes Amazon look very attractive. It largely depends on idle time however - and we in any case would not go without an in house cluster for testing and development. It also appears we now may need to process 500 Million sources to counteract possible spatial error correlations (as presented by Berry Holl in Heidelberg in 2009). This would bring the saving close to zero - but one must question if the likes of Amazon can do a cheaper job of maintaining a cluster.

The availability of a much larger number of nodes than we can buy is very interesting both for testing and for production. By using Amazon we could perhaps do our processing faster by using more nodes and still have it cost less than an in house machine. We shall continue to experiment and evaluate this option - in any case the final machine at ESAC for the processing will not be purchased until we are a couple of years into the mission around 2015 .

\subsection{Virtualisation - non uniqueness}

The real power of Java comes from the notion of running on a virtual machine. This should not be, but often is, confused with interpretation such as done in LISP and Smalltalk. This virtue is not shared by many new languages or schemes perhaps the most all encompassing of which is the Microsoft .NET framework in which many languages are compiled to the same virtual machine. We are not trying to say Java is the only language with this feature. In fact with virtualisation suddenly any particular machine/OS/langugae configuration can be equally portable with its own virtual machine. Suddenly putting those legacy apps in a cloud may not be such a hard decision.

\subsection{Future of Java}

The rise of Java in industry and positive experience we had we Java compared to C++ reinforced our choice of Java as the language for Gaia. This choice was reaffirmed within the Gaia community in 2006 (Hernandez et al, 2006). Indeed at the time of writing all science development missions at ESAC are using Java as their programming language.

We predict that Java will be the language of choice for the foreseeable future because of all the advantages outlined earlier, although we believe that within the Java Virtual Machine, we will see more use of dynamic languages such as Ruby and Groovy for areas of the application that will need to be changed very frequently.

\section{Conclusion}

One may argue about the definition of High Performance Computing but within the Gaia project since the late 90 s we have certainly be doing numerically intensive computing and handling increasingly large amounts of data in a highly distributed manner. All of this is done using Java. We argue that this is a good option for long running projects where portability and maintenance are possibly more important than squeezing the last FLOP from a specific processor.

The portability of Java has served us well in the last decade allowing code to move from SPARC station to Linux boxes, WinTel and even the Marenostrum super computer. Of late we have also leveraged Amazon's E2C resources. 
We are not alone in the astronomy world, many other projects are using Java or other high level languages. The ESA missions Herschel, Planck and many archives are Java users. Others such as Spitzer and JWST mentioned using Java at least in part. Other higher level languages such as $\mathrm{C \#}$ and Python are also in use. 


\section{A Acronyms used in this paper}

The following table has been generated from the on-line Gaia acronym list:

\begin{tabular}{|l|l|}
\hline Acronym & Description \\
\hline ADASS & Astronomical Data Analysis Software and Systems \\
\hline AGIS & Astrometric Global Iterative Solution \\
\hline BSC & Barcelona Supercomputing Centre \\
\hline CERN & Centre Europénne pour la Recherche Nucléaire \\
\hline CESCA & CEntre de Supercomputacío de CAtalunya \\
\hline CNES & Centre National d'Etudes Spatiales (France) \\
\hline CPU & Central Processing Unit \\
\hline DPAC & Data Processing and Analysis Consortium \\
\hline DPACE & Data Processing and Analysis Consortium Executive \\
\hline ESA & European Space Agency \\
\hline ESAC & European Space Astronomy Centre (VilSpa) \\
\hline FLOP & FLoating-point OPeration \\
\hline GB & GigaByte \\
\hline HPC & High-Performance Computing \\
\hline ICRS & International Celestial Reference System \\
\hline IEEE & Institute of Electrical and Electronic Engineers \\
\hline JIT & Just In Time Compiler \\
\hline JNI & Java Native Interface \\
\hline JVM & Java Virtual Machine \\
\hline JWST & James Webb Space Telescope (formerly known as NGST) \\
\hline MB & MegaByte \\
\hline OO & Object Oriented \\
\hline PMD & Software tool to detect software problems \\
\hline RAM & Random Access Memory \\
\hline RMI & Remote Method Invocation \\
\hline SAN & Storage Area Network \\
\hline SOC & Science Operations Centre \\
\hline TB & TeraByte \\
\hline UB & University of Barcelona (Spain) \\
\hline UML & Unified Modeling Language \\
\hline VM & Virtual Machine \\
\hline
\end{tabular}




\section{B Bibliography}

\section{References}

Bombrun A, Lindegren L, Holl B, Jordan S (2010) Complexity of the Gaia astrometric leastsquares problem and the (non-)feasibility of a direct solution method. aap 516:A77, DOI 10.1051/0004-6361/200913503OPEN:

ESA (1997) The Hipparcos and Tycho Catalogues. ESA, eSA SP-1200

FP JB (1987) No silver bullet essence and accidents of software engineering. Computer 20:1019, DOI http://doi.ieeecomputersociety.org/10.1109/MC.1987.1663532

Hernandez J, O'Mullane W, Huc C (2006) Language recommendation for the DPAC. Tech. rep., European Space Astronomy Centre, Villafranca, Madrid, URL http://www.rssd.esa.int/llink/livelink/open/532588

Kahan W, Darcy J (1998) How java's floating-point hurts everyone everywhere. Tech. rep., Elect. Eng. \& Computer Science, University of California at Berkely, keynote presented at ACM 1998 Workshop on Java for High-Performance Network Computing held at Standford University, http://www.cs.berkeley.edu/ wkahan/JAVAhurt.pdf

Klioner SA (2003) A Practical Relativistic Model for Microarcsecond Astrometry in Space. Astrophysical Journal 125:1580-1597

Lindegren L, O'Mullane W, Lammers U, Hobbs D (2008) The astrometric core solution for the gaia mission. in prepreation for Astronomy and Astrophysics

Makarov VV (1998) Absolute measurements of trigonometric parallaxes with astrometric satellites. Astronomy and Astrophysics 340:309-314

Murray CA (1983) Vectorial astrometry. Bristol: Adam Hilger, 1983

O’Mullane W, Lindegren L (1999) An Object-Oriented Framework for GAIA Data Processing. Baltic Astronomy 8:57-72

O’Mullane W, Hazell A, Bennett K, Bartelmann M, Vuerli C (2000) ESA Survey Missions and Global Processing. In: Manset N, Veillet C, Crabtree D (eds) Astronomical Data Analysis Software and Systems IX, Astronomical Society of the Pacific Conference Series, vol 216, pp 419-422

O'Mullane W, Lammers U, Bailer-Jones C, Bastian U, Brown AGA, Drimmel R, Eyer L, Huc C, Katz D, Lindegren L, Pourbaix D, Luri X, Torra J, Mignard F, van Leeuwen F (2007) Gaia Data Processing Architecture. In: Shaw RA, Hill F, Bell DJ (eds) Astronomical Data Analysis Software and Systems XVI, Astronomical Society of the Pacific Conference Series, vol 376, pp 99-108

O'Mullane W, Lindegren L, Lammers U, Hobbs D (2008) Implementation of the gaia astrometric global iterative solution in java. in prepreation for Experimental Astronomy

Perryman MAC, de Boer KS, Gilmore G, Høg E, Lattanzi MG, Lindegren L, Luri X, Mignard F, Pace O, de Zeeuw PT (2001) GAIA: Composition, formation and evolution of the Galaxy. Astronomy and Astrophysics 369:339-363, DOI 10.1051/0004-6361:20010085, arXiv:astro-ph/0101235

Shiers J (2009) Grid today, clouds on the horizon. Computer Physics Communications 180(4):559 - 563, DOI DOI:10.1016/j.cpc.2008.11.027, URL http://www.sciencedirect.com/science/article/B6TJ5-4V70NH3-1/2/2fdc77415de62c2e360754c969911850, special issue based on the Conference on Computational Physics 2008 - CCP 2008

Wieprecht E, Brumfit J, Bakker J, de Candussio N, Guest S, Huygen R, de Jonge A, Matthieu JJ, Osterhage S, Ott S, Siddiqui H, Vandenbussche B, de Meester W, Wetzstein M, Wiezorrek E, Zaal P (2004) The HERSCHEL/PACS Common Software System as Data Reduction System. In: Ochsenbein F, Allen MG, Egret D (eds) Astronomical Data Analysis Software and Systems (ADASS) XIII, Astronomical Society of the Pacific Conference Series, vol 314, pp 376-379 